\title{
LA 'DONNA ANGELICATA' O LA DESHUMANIZACIÓN \\ DE LA MUJER: APROXIMACIÓN AL CONCEPTO DE MUSA EN EL DOLCE STIL NOVO ${ }^{1}$
}

\author{
Antonia Víñez Sánchez ${ }^{2}$ \& Inmaculada Concepción Víñez Daza ${ }^{3}$ \\ Universidad de Cádiz
}

Fecha de recepción: 09/06/2021

Fecha de aceptación: 31/10/2021

\begin{abstract}
Resumen
En los albores del Humanismo, ya desde el "otoño de la Edad Media", se diseña un concepto de musa cuya lectura es fuente de perfección, símbolo de belleza suprema e inalcanzable para el artista y causa de su sufrimiento constante. La imagen exterior se corresponde con un ideal de mujer espiritualizada al extremo, paradójicamente víctima de una divinización que la aparta definitivamente de cualquier dimensión humana. Este trabajo reflexiona sobre los inconvenientes de ser "musa" en la transición de la Baja Edad Media al Renacimiento, centrándose en el corpus de la escuela poética del Dolce Stil Novo.
\end{abstract}

Palabras clave: musa, donna angelicata, Humanismo, Dolce Stil Novo.

\begin{abstract}
At the dawn of Humanism, as early as the "waning of the Middle Ages", a new concept of muse is formulated as source of perfection and symbol of supreme beauty - so much so, that it is unattainable by artists, causing them constant suffering. The external image that develops is that of a woman spiritualized to the extreme, victim of a deification that removes her from any human dimension, which is in itself paradoxical. In this paper I examine the drawbacks of being a "muse" during the transition from the late Middle Ages to the Renaissance, focusing on the texts of the poetical school of the Dolce Stil Novo.
\end{abstract}

Keywords: muse, donna angelicata, Humanism, Dolce Stil Novo.

\footnotetext{
${ }^{1}$ Investigación financiada por el Grupo de Investigación HUM725 de la Junta de Andalucía (LELIROM) con sede en la Universidad de Cádiz.

2 Profesora Titular de Universidad, Departamento de Filología, Universidad de Cádiz (España); antonia.vinguez@uca.es

${ }^{3}$ Investigadora del Grupo de investigación HUM725 de la Junta de Andalucía, Departamento de Filología, Universidad de Cádiz (España); inma.vinezdaza@alum.uca.es

Raudem, Revista de Estudios de las Mujeres. Vol. 9, 2021. ISSN: 2340-9630
}

https://doi.org/10.25115/raudem.v9i1.5528

(C) Publicado bajo licencia Creative Commons BY-NC-ND 4.0 
El concepto de musa, procedente como sabemos de la mitología y deidades de índole femenina, ha sido abordado como arquetipo universal e interpretado en su acepción simbólica como sinónimo de inspiración. De hecho, ambos términos suelen hallarse en continua sustitución uno del otro de forma reiterativa. El campo semántico que rodea al concepto gira en torno a nociones que pueden resumirse en un triángulo de cualidades presentes de modo recurrente: belleza, sabiduría e inocencia. Así, el arquetipo de la musa constituye el eje central en la poesía amorosa y esta vinculación deriva, irremediablemente, en el hecho de que "es la fuerza inspiradora más enérgica de la poesía", como señala M. Dunn Mascetti (1990: 59).

Tras la herencia de la poesía cortés, que desarrolló por toda la geografía europea un modo de amar conceptuado y reglamentado -la fin'amors- en el que la dama-Midonsse erigía epicentro de sentimiento y comportamiento social, llegando a constituir una gineolatría obsesiva (Salinas 1981: 25), el "otoño de la Edad Media" introduce un entorno de pensamiento humanista, infraestructura del Renacimiento, en el que se consolidará un universo cuyo eje sigue siendo femenino, si bien éste merece una lectura crítica y atenta. La expresión, estandarizada, como sabemos, a partir del imprescindible estudio de $\mathrm{J}$. Huizinga, se centra en los siglos de la Baja Edad Media, en los cuales "se abandonó de nuevo el sistema del amor cortés, tan artificiosamente construido" en aras de una estilización del amor (1985: 153-155).

Algunas manifestaciones del perfil de la Domna trazado por los trovadores, combatido frontalmente por algunas trobairitz, como es el caso de Castelloza y La Comtessa de Dia, permea en la escuela poética del Dolce Stil Novo, cuyo marbete identificador anuncia una "novedad" que puede aplicarse también a la noción de musa. El conjunto del corpus poético representa el anhelo humano de amor perfecto, de fusión de almas, a partir de un proceso de idealización extremo que va a transformar la conceptualización del amor enalteciendo a la nueva dama, elevándola a una esfera inexpugnable, espiritualizándola al límite para compartir el espacio que la posicionará junto a la Virgen en lo más alto del Paraíso, tal como Dante Alighieri relata ${ }^{4}$. Es un proceso complejo, idealizado asimismo por la literatura crítica, que exige una revisión conforme a la óptica de la socioliteratura y cuyas consecuencias son tan sutiles, que resultan paradójicas ${ }^{5}$.

\footnotetext{
${ }^{4}$ En la Divina Comedia Dante describe a Beatriz sentada en la tercera grada de la Virgen María, c. XXXII, vv. 7-9 (González Ruiz 1980: 524)

${ }^{5}$ Para el enfoque histórico-social de la obra literaria, desarrollado sobre todo en la Escuela de Tartu, cf. Pozuelo Yvancos y la bibliografía ahí referida (1988: 66-74).
} 
De este modo, y centrándonos en el periodo cronológico acotado, El Dolce Stil Novo constituye el foco de esta novedad inquietante. Ubicado en el periodo histórico conocido como "Baja Edad Media", resulta un cruce de caminos cuya expresión es fusión de tradición y originalidad. La nueva musa será el resultado de este proceso de miscelánea cultural en el marco de una definición flexible de "Humanismo" y teniendo presente que el concepto, en sí mismo, es adaptable a momentos concretos de la cultura europea del alto medievo, como el denominado "humanismo chartrense" del siglo XII, con el intelectual Abelardo a la cabeza (Le Goff 1990: 57-64) y, sobre todo, con el protagonismo de Eloísa, pero también puede aplicarse al "espíritu enciclopédico" del siglo XIII (Vàrvaro 1983: 35), que sirvió de plataforma al estallido humanista de finales de ese mismo siglo con la escuela poética estilnovista.

Lleno de matices y singularidades, producto de la introspección, camino ya del individualismo poético que cristalizará en la figura de Francesco Petrarca, el Dolce Stil Novo surge de la necesidad de hallar cauces de expresión lírica alejados del feudalismo caduco, por lo que su escenario es la Italia de los comune, expresión de un nuevo orden social urbano y generador del protagonismo de una aristocracia burguesa intelectual -el civitas burgués- que decide los destinos políticos y culturales de los diferentes estados que la componen.

Una figura de gran relieve como Guido Guinizzelli, maestro y guía de la escuela, supondrá la síntesis de los valores corteses que la poesía siciliana hereda y la causalidad doctrinal de la poesía toscana. El personaje, identificado como Guinizzelli da Magnano, nace en la Bolonia del Duecento hacia 1216/1217 (Rossi 2004: 27). Exiliado en 1274 tras la victoria güelfa, por pertenecer a la facción gibelina, muere el 14 de noviembre de 1276 (Contini 1960 II: 447). Su obra representa una síntesis conceptual de la fuerte influencia del toscano Guitonne d'Arezzo, del heterodoxo franciscanismo reinante en el norte de Italia y de la poesía siciliana, ya que entra en contacto con los poetas del entorno del rey Enzo, hijo de Federico II, que fue hecho prisionero en Bolonia en 1249 (Dronke 1995: 197).

Si partimos de la noción de que la Midons de la poesía trovadoresca es, como la definió J. E. Ruiz Doménec, "la mujer que mira”, este hecho implica que la ficción poética es, al mismo tiempo, la plasmación de la realidad en el contexto cortés de los siglos XII y XIII: "La generación de ideas en el interior de una sociedad -la idealización- es, en sentido estricto, una aceptación pasiva de la realidad vigente, a la que tan sólo se matiza o se suaviza (se sublima, diría Freud) mediante criterios de enmascaramiento o 
falsificación que conduce en ocasiones a lo cursi". No olvidemos que "la moral matrimonial ya se ha instalado definitivamente en el interior de la buena sociedad", pero tampoco que "las mujeres buscan su «reconformación» en una sexualidad abierta, extramatrimonial” (1990: 20-23). Así lo explicaba Andrés el Capellán en su famoso tratado De Amore en el siglo XII: "Causa coniugii ab amore non est excusatio recta" (Creixell 1985: 362). Si acudimos al testimonio de las propias mujeres, escuchando su voz a través de sus manifestaciones poéticas, hallamos afirmaciones tan contundentes como las de algunas trobairitz.

En el ideario amoroso de la cansó, la mujer, como destinataria del mensaje poético, se sitúa en una posición de superioridad a imitación del vínculo feudo-vasallático, pues el reglamento cortés exige un poeta-vasallo, emisor del texto. Sin embargo, esta sencilla fórmula se complica cuando los papeles se invierten en las composiciones de las trobairitz, ya que en las últimas décadas se ha empezado a comprender la visión particularmente crítica que algunos textos reflejan acerca de los comportamientos convencionales, lo que supone una modalidad diferente de superioridad femenina y la denuncia de fisuras en la conceptualización del amor (Víñez 2017: 271-272). Así sucede, por ejemplo, en la cansó Amics, s'ie·us trobes avinen de Castelloza (Rieger 1991: 519520), trobairitz de principios del siglo XIII, cuya escueta vida la cataloga de "gentils domna” (M. de Riquer 1983: III, 1325-1326). El resumen de M. Martinengo sintetiza la intención del texto (1997: 88-91): "En esta canción, la poeta muestra gran repugnancia hacia la hipocresía y el engaño y lanza un desafío a las costumbres: dicen que no es conveniente que una mujer corteje a un caballero y, sin embargo, yo lo hago". En consecuencia, desde los primeros estudiosos, se ha repetido en innumerables ocasiones el rasgo de la "sinceridad" de los textos femeninos corteses, observados con una mirada no exenta de moralismo, que insiste en el carácter nada pudoroso de algunas trobairitiz como La Comtessa de Dia, cuyo lenguaje cataloga Coll i Vehí de "ardiente” en 1861, como recuerda I. de Riquer (1997: 33-34).

Meg Bogin, en su emblemático estudio Les femmes troubadours, publicado en New York en 1976, defendió el protofeminismo de estas poetas: "Ensuite, bien qu'elles traitent de l'amour, le langage des femmes et les situations qu'elles exposent diffèrent singulièrement de ceux de leur émules masculins" (1978: 15). La singularidad de buena parte de sus composiciones dota a la mujer trovadora de un sentido activo determinante en la relación poético-amatoria. Las trobairitz asumen el código lingüístico feudal del amor cortesano, pero no se inhiben en la especulación mental sexual, más propia de la 
poesía masculina -recordemos el amor de loing ("amor de lejos") de Jaufré Rudel, por ejemplo-, por lo que desautomatizan el tópico del grado drutz sin dar cabida a la duda. Así podemos apreciarlo en el caso de la tensó entre la desconocida Domna H y Rofin (Rieger 1991: 292-294), que supone un desacato a las normas de la cortesía, con el empleo de un lenguaje explícito por medio del trovar leu, sin ambigüedades. El texto dirime la cuestión de si se comporta mejor un caballero que decide romper las normas de cortesía alcanzando la relación sexual con su dama o el que prefiere acatarlas y mantiene la distancia. La dama $\mathrm{H}$-enigmática denominación por medio de una inicial que encubre una identidad hasta ahora no averiguada- prefiere al caballero que rompe el pacto consumando la relación sexual plena:

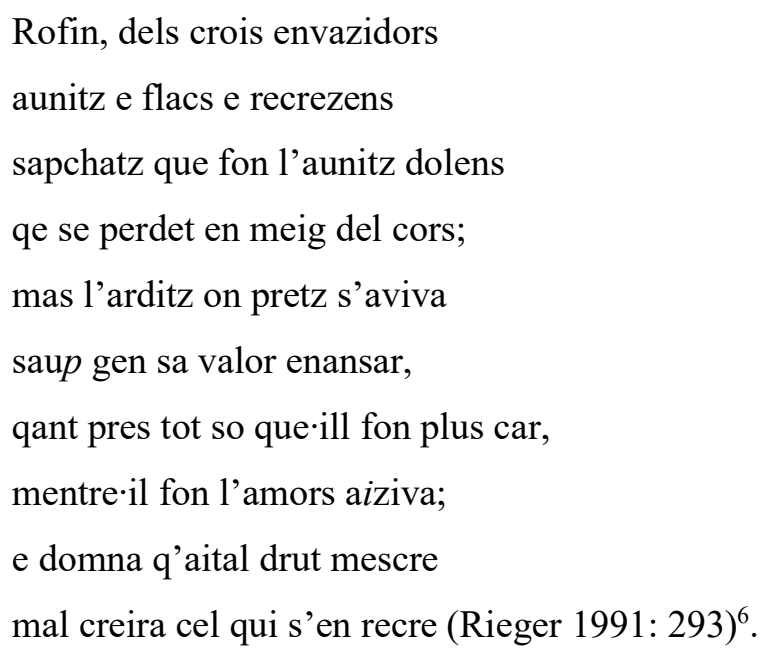

También la cansó Estat ai en greu cossirier de La Comtessa de Dia (Rieger 1991: 600601) manifiesta el deseo explícito de la trobairitz de tener al caballero desnudo en sus brazos (Víñez 2013: 55-57):
Ben volria mon cavallier
tener un ser en mos bratz nut
q'el s'en tengra per ereubut
sol q'a lui fezes consseillier" (Rieger 1991: 600)7.

\footnotetext{
6 "Rosin, sabed que el triste deshonrado / que se perdió en medio del camino / forma parte de los malvados perturbadores, / desvergonzados, viles y cobardes, / mientras el atrevido en quien el valor se reaviva / notablemente supo enaltecer su valor, / cuando tomó todo lo que más quería / en el momento en que el amor le estaba más disponible / y la dama que rechaza a un amante así, / mal repondrá su confianza en aquel que no se atreve" (Trad. Martinengo 1997: 121-123).

${ }^{7}$ Cómo querría una tarde tener / a mi caballero, desnudo, entre los brazos, / y que él se considerase feliz / con que solo le hiciese de almohada" (Trad. Martinengo 1997: 62).
} 
En todos estos casos, el hombre-musa era requerido de forma manifiesta para la consumación sexual, más allá de la idealización amorosa. Pero cuando llegamos a la famosa canzone del poeta boloñés Guido Guinizzelli Al cor gentil rempaira sempre amore, apreciamos el giro hacia una nueva estética poética que se aleja de los elementos feudales de la fin'amors, evocados por Guittone d'Arezzo, y que ya no tienen la misma significación. El racionalismo escolástico que representa el segundo era liberado hacia una metafísica de la "donna angelo", creación, como pensamos, del boloñés (Víñez, 2016: 674). Para ello, el poeta diseña el precedente de la dama angelical: "la metafora della donna-stella-afirma I. Bertelli-si presenta come un motivo veramente emblematico nella poesia del Guinizzelli” (1983: 199 [1]), y esa "dama-estrella” es el sustento metafísico de la donna angelicata, expresión de omnímoda idealización. Como dice C. Alvar, "el poeta reelabora el recuerdo y reflexiona sobre sus propios sentimientos, por lo que resulta imposible distinguir la realidad de la ficción, la dama y la idea" (1984: 11).

En este punto, es necesario repensar la configuración de la musa poética en los nuevos términos expresados por el inspirador del estilnovismo y matizar su evolución en los continuadores de la escuela a partir de la herencia cortés: "La mujer, como escribe Cohen, -nos describía P. Salinas- es siempre superior, inaccesible al poeta trovador (...). Abajo el que canta y suspira, y arriba aquella apenas entrevista hermosura de frente velada. Se adelanta día a día por el camino de la sublimación, la divinización de esa mujer que es y no es, que se escapa, por sus propias perfecciones en esta tierra, del mundo, y que terminará en la donna angelicata” (1981: 25-26).

Al cor gentil, poema emblemático, fabrica la "donna angelo", como expresan sus conocidos versos finales (58-59): "Tenne d'angel sembianza/ che fosse del Tuo regno" (Pelosi 1998: 42-43) ${ }^{8}$. La crítica, como es ya habitual al hablar de Guinizzelli, no haya acuerdo en establecer el sentido de esta imagen, pero es evidente, con todo, que evoluciona de un uso decorativo en la poesía cortés y posteriormente en la siciliana, a un sentido simbólico en el boloñés, huyendo de evocar a la mujer real. Aunque el poema continúa la tradición de la poesía occitana del análisis psicológico del sentimiento amoroso, la principal novedad que la canzone aporta es el nuevo ideal de gentilezza, o nobleza del corazón. A esto se añade un nuevo perfil de dama-musa muy mediatizada ya por los valores asentados de la iglesia, gran triunfadora de la Baja Edad Media en la conceptualización de la nueva dama, resultado de las ideas humanistas acerca del

\footnotetext{
8 “Parecía ángel / de Tu reino" (Trad. Alvar 1984: 21).
} 
matrimonio, que difunden el concepto de mujer-procreadora, centrada en la dedicación a las tareas domésticas, como un retroceso evidente a los logros puntuales de las trobairitz, portavoces de la sexualidad libre y elegida voluntariamente fuera del matrimonio. Como puntualiza R. de Maio (1988: 227-232), una imagen contraria a la de siglos anteriores: “en otros tiempos Eloísa había sostenido indemne el amor libre, para no impedir con el matrimonio el destino de Abelardo. Pero entonces no existía un Santo Oficio Romano, ni había habido un Concilio de Trento" (1988: 101). Eloísa, siempre a la sombra del filósofo y de la que apenas tenemos datos biográficos, había manifestado su firme determinación:

Dios sabe que nunca busqué en ti nada más que a ti mismo. Te quería simplemente a ti, no a tus cosas. No esperaba los beneficios del matrimonio, ni dote alguna. Finalmente, nunca busqué satisfacer mis caprichos y deseos, sino -como tú sabeslos tuyos. El nombre de esposa parece ser más santo y más vinculante, pero para mí la palabra más dulce es la de amiga y, si no te molesta, la de concubina o meretriz (Santidrián y Astruga 1993: 104-105).

La mujer, reafirmándose en su debilidad, heredada de siglos anteriores y fundamentada en la pseudociencia etimológica, tal como Isidoro de Sevilla había sustentado ${ }^{9}$, es instrumento al servicio del mundo. El espejo en que debe mirarse es la virgen María en su resignada humildad, sin méritos propios, cuya obligación es iluminar, ser guía, en completo silencio como rechazo a la vida mundana, según reflejan los tratados pedagógicos y su ideario de mujer doméstica. Por ello, no extraña encontrar en el poema inaugural del Dolce Stil Novo la aseveración siguiente:

così dar dovria, al vero,

la bella donna, poi che'n gli occhi splende

del suo gentil, talento

che mai di lei obedir non si disprende (Pelosi 1998: 41-42) ${ }^{10}$.

El resto de la escuela, en su afán por formatear a la nueva musa angelical, se concentra más en la definición del dios Amor, como ente abstracto; así sucede en Guido Cavalcanti, nacido hacia 1260 en Florencia, de donde fue desterrado por los priores - entre otros, por

\footnotetext{
${ }^{9}$ En las Etimologías aparece el término "mullier" asociado a "mollities" (debilidad, blandura) (Oroz Reta y Marcos Casquero 2004).

10 “Así debería recompensar / la bella dama a su fiel servidor, / pues desde el momento en que brilla a sus ojos / no tiene otro deseo que obedecerle" (Trad. Alvar 1984: 19).
} 
el que había sido su amigo, Dante- muriendo en el verano de 1300 (Contini 1960, 2_II: 487-490). A pesar de la heterodoxia de su pensamiento, de tintes averroístas, en su texto más conocido, Donna me prega, per ch'eo voglio dire, se produce este desplazamiento:

Donna me prega, - per ch'eo voglio dire

d'un accidente- che sovente - è fero

ed è sì altero -ch'è chiamato amore (Contini 1960, 2-II: 522-523) ${ }^{11}$.

Todo el poema es un tratado de amor en verso, más filosófico que lírico propiamente, e irradia un neoplatonismo evidente: el intelecto no puede producir la pasión pero sí puede contemplar el bien (estrofa II). Centrado asimismo en la sintomatología amorosa (estrofa III), propondrá que sólo del amor incorpóreo puede obtenerse recompensa (estrofa V). Su discípulo, Dino Frescobaldi, que vivió entre 1271 y 1316 (Contini 1960, 2-II: 615), incide en el sufrimiento de amor de corte neoplatónico, dignificando a la dama al extremo:

Quest'è la donna piena di merzede,

in cui ogni vertù bella si fida.

Vienle dinanzi Amor che par che rida,

mostrando'l gran valor dov'ella siede;

e quando giunge ov'Umiltà la chiede,

par che per lei ogni vizio s'uccida (Marti 1969: 357-358) (2. $^{12}$

Con tintes muy guinizzellianos, también se dirige a una musa desdeñosa y cruel que es causa de un sufrimiento extremo que merma su vida:

Un'alta stella di nova bellezza,

che del sol ci to'l'ombra la sua luce,

nel ciel d'Amor di tanta virtù luce,

che m'innamora de la sua chiarezza.

( ...)

E come donna questa nova stella

sembianti fa che ' 1 mi'viver le spiace

\footnotetext{
11 "Porque me lo ruega una dama, quiero hablar de un accidente que a menudo es cruel y despiadado, y que se llama amor" (Trad. Alvar 1984: 39).

12 "Ésta es la dama llena de mercedes, / en quien se asientan todas las virtudes. / Sonriente le viene Amor delante / mostrándole el alto puesto que ocupa; / cuando llega a donde Humildad la llama, / parece que ella mata a los vicios" (Trad. Alvar 1984: 111). La humildad, como señalamos más arriba, es una virtud muy estimada en todo el estilnovismo ya que forma parte del paradigma virginal.
} 
e per disdegno cotanto è salita (Marti 1969: 359-360) ${ }^{13}$.

Y en otro soneto, sentencia: "Merzé, donna crudel, giovane e bella!" (Marti 1969: 364) (4 $^{14}$ concentrando en un solo verso el arquetipo triunfante de la mujer como causa de la infelicidad del poeta.

Cino da Pistoia es el poeta más humanizado y original de la escuela estilnovista, si exceptuamos a Dante, que trascendió los límites de la misma y se convirtió en poeta inclasificable, como la mayor parte de su obra amorosa. El pistoyés, nacido hacia 1270, jurista afamado, muere a finales de 1336 o principios del año siguiente (Contini 1960: 2II: 629-630). Realmente, es más que un eslabón entre Dante y Petrarca, con lugar propio, derivado del hecho de su personalísima expresión más allá de la retórica algo manida ya de Frescobaldi: "aleja de sus versos -afirma Alvar- las preocupaciones metafísicas y los llena de vivencias personales, profundamente auténticas" (1984: 121). El corazón rezuma deseo, como sede del amor que es, a partir de la contemplación de los ojos. El hermoso rostro de la dama nos perfila, aunque sea de lejos, una presencia real, casi intuida:
Veduto han gli occhi miei sì bella cosa,
che dentro dal mio cor dipinta l'hanno,
e se per veder lei tuttor no stanno,
infin che non la trovan non han posa (Marti 1969: 431) ${ }^{15}$.

De este modo, el poeta recupera la antigua costumbre de las damas de compañía -que tanto éxito tendrán con Dante-, y solicita que sean intermediarias, denunciando la ausencia material de la dama, que no ha acudido a una fiesta, convirtiendo en poético lo anecdótico:
Come non è con voi a questa festa,
donne gentili, lo bel viso adorno? (...)
Eo vi prego, donne, sol per Deo;
se non volete ch'io di ciò mi moia,

\footnotetext{
13 "Una alta estrella de nueva belleza / cuya luz quita la sombra al sol, / en el cielo de Amor brilla tanto / que me enamora con su claridad. (...) Y como dama, esta nueva estrella / muestra desagrado por mi vivir / y así, con desdén, se eleva distante" (Trad. Alvar 1984: 113).

14 “ ¡Compasión, dama cruel, joven y hermosa!” (Trad. Alvar 1984: 117).

15 "Han visto mis ojos tal hermosura / que la han pintado en mi corazón / y así para verla no se detienen / y hasta que no la encuentren no descansan" (Trad. Alvar 1984: 123).
} 
fate sì che stasera la vegg'eo (Marti 1969: 445-446) ${ }^{16}$.

Sin renunciar al deseo de contacto físico con la musa, Cino da Pistoia es víctima de la "novedad" que supone la donna angelicata:

Guardando a voi, in parlare e'n sembianti

angelica figura mi parete,

ché sopra ciascum mortal cor tenete

compimenti di ben non so dir quanti (Marti 1969: 449) ${ }^{17}$.

El ideal de dolcezza dibuja un nuevo estilo que aleja cada vez más a la musa de la mujer real que habitó en los poemas occitanos, a veces sólo como receptora, con las dificultades de un diseño preconcebido, pero otras manifestando en primera persona sus anhelos y deseos, en ocasiones muy físicos y terrenales, como era el caso de las trobairitz referidas más arriba.

La fusión del artista y su numen va a encontrar expresión sublime en Beatriz, culminación del boceto de la mujer-guía. Ya desde sus primeros sonetos, Dante, que había nacido en Florencia en mayo de 1265 y adquirió sus conocimientos de corte neoplatónico en la Universidad de Bolonia, relata en la Vita Nuova el desarrollo de estos nuevos valores poéticos: "suprime cualquier censura de la dama: la amada es enviada de Dios, es un ángel -deuda con Guinizzelli- dispuesta a abandonar este mundo lo antes posible y, naturalmente, muere joven", como señala Alvar (1984: 70). La dama se vuelve más inefable que nunca:

De gli occhi de la mia donna si move

un lume sí gentil che, dove appare,

si veggion cose ch'uom non pò ritrare

per loro altezza e per lor esser nove (Pirovano y Grimaldi 2015: 739-740) ${ }^{18}$.

La misión de guía, que es atributo prioritario de la musa, va unida a su asimilación a la virgen en Di donne io vidi una gentile schiera:

16 “¿Cómo no está con vos en esta fiesta, / nobles damas, el más hermoso rostro? (...) Os ruego, señoras, sólo por Dios, / si no queréis que pronto me muera, / que hagáis que pueda verla esta tarde" (Trad. Alvar 1984: 125).

17 “Al veros, en el hablar y en el rostro / me parecéis angélica figura; / sobre cualquier corazón mortal tenéis / incontables virtudes, no sé cuántas" (Trad. Alvar 1984: 127).

18 "De los ojos de mi dama procede / tan noble luz que cuando aparece / se ven cosas que nadie contaría / por lo elevadas y porque son nuevas" (Trad. Alvar 1984: 73). 
Credo che de lo ciel fosse soprana,

e venne in terra per nostra salute (Pirovano y Grimaldi 2015: 785) ${ }^{19}$.

En el Paraíso (canto I, vv. 100-102), Dante presenta una imagen asexuada de Beatriz y describe un sentimiento filio-maternal entre ambos:

Ond'ella, appresso d'un pio sospiro,

li occhi drizzò ver me con quel sembiante

che madre fa sovra figlio deliro (González Ruiz 1980: 367) ${ }^{20}$.

La poesía es, por otro lado, introspección. Es un análisis concienzudo de los efectos del amor, interiorizados hasta el punto de que el poeta estilnovista se desgrana a sí mismo, fragmentándose en matices del sentimiento amoroso y centrando el discurso, en consecuencia, más en su trasfondo emocional que en la dama, como antesala de la devoción del poeta a su ego, que protagonizará Francesco Petrarca unas décadas más tarde con su Canzoniere y con los Trionfi. Esto sucede antes en Voi, donne, che pietoso atto mostrate de Dante:

Ma se tu mirerai il gentil atto

de li occhi suoi, conosceraila poi:

non pianger piú, tu sè già tutto sfatto (Pirovano y Grimaldi 2015: 792-793) ${ }^{21}$.

Ahora bien, lo que sabemos de la dama-guía, esa nueva donna angelicata, es producto de la figuración del poeta-hombre. Como afirma Boyer acerca del discurso misógino, "su presencia es tan universal que probablemente subyace incluso en géneros que idealizan a la mujer poniéndola en un pedestal, como hacen el amor cortés con la domna, el culto mariano con la Virgen o el dolce stil novo con la donna angelicata” (1989: 260).

Sin embargo, no hemos de esperar a la irrupción de las poetas renacentistas con voz propia, ya a finales del siglo XV y principios del XVI, con figuras como Verónica Gámbara (nacida en 1485), Vittoria Colonna (en 1490), Laura Terracina (en 1510), Chiara Matraini (en 1514), Laura Batiferri (en 1523) y Gaspara Stampa (mismo año), entre otras,

\footnotetext{
19 "Creo que del cielo era soberana / y bajó a la tierra para salvarnos" (Trad. Alvar 1984: 75).

20 “A lo que ella, después de suspirar piadosamente y dirigiendo los ojos hacia mí con aquel semblante que pone la madre ante los extravíos del hijo, replicó” (Trad. González Ruiz, íbidem).

21 "Pero si tú contemplas la nobleza / de sus ojos, la reconocerás: / no sigas llorando, que estás deshecho" (Trad. Alvar 1984: 77).
} 
para oír a las mujeres de letras. En medio, la literatura femenina halla exponentes tan relevantes como la renombrada Christine de Pizan (1365-1431): "Las poetisas italianas, por tanto, no son una floración espontánea, sino las herederas de una larguísima tradición, a las que el amplio desarrollo de la imprenta en Italia, y una mayor consideración general por la tarea intelectual y artística de la mujer, pone en óptimas condiciones para encontrar eco y aplauso para su obra”, como señala L. Martínez de Merlo (1988: 11).

M. Arriaga ha visibilizado voces de mujeres silenciadas en este desierto de casi dos siglos señalando que "mientras que los poetas del Dolce Stil Novo pasan a la historia como una generación compuesta por muchos poetas, las poetas (pero también las prosistas místicas), protagonizan un caso de cultura silenciada". Todas estas mujeres de letras de los siglos XIII y XIV han sido cuestionadas -como las trobairitz- hasta el punto de que se dude sobre su existencia. Autoras como Compiuta Donzella (1250), Nina Siciliana (1290), Selvaggia dei Vergiolesi (1308), Giovanna Bianchetti (1310), Ortensia de Guglielmo (1350), Giustina Levi Perotti (1350), Bartolomea Mattugliani (1350), Leonora della Genga (1360), Livia del Chiavello (1380) y Elisabetta Trebbiani (1397) "pueden considerarse el primer grupo literario compuesto por mujeres en Italia". Son mujeres poetas vinculadas al movimiento místico europeo que expresan su disconformidad con el sistema espiritual pero también con la sociedad laica. "La ausencia de mujeres poetas en el siglo XIII - continúa Arriaga- contrasta con la presencia de escritoras en prosa (...) y con la de eruditas ligadas a las universidades" (2012: 7-10). La trascendencia de su presencia en estos círculos intelectuales es innegable, pero no lo es menos que los testimonios de las primeras poetas -Nina Siciliana y Compiuta Donzellacuestionan el servicio amoroso desde la sinceridad poética (Arriaga, 2012: 29-48) anticipándose, como ya hicieron las trobairitz, al movimiento de la "Querelle des femmes" de Chritine de Pizan (Víñez y Sáez, 2018: 11-26).

Ante esta panorámica, la pregunta que ha de formularse es si la musa que los poetas del Dolce Stil Novo dibujan representa los deseos de la mujer de su tiempo. No hay duda acerca de la cultura misógina que reina en occidente a lo largo de los siglos medievales y que se propaga al Renacimiento que, como afirma R. De Maio hubiera podido hacer más por las mujeres (1988: 7). Es la razón de que O. Niccoli se pregunte si realmente hubo un renacimiento para éstas (1993: 12-13). Aun así, no es baladí el esfuerzo que supuso la remontada de estas escritoras nadando contra corriente en un momento poético como es el Dolce Stil Novo que, desde la óptica del contexto cultural reinante, se esforzó por trazar un retrato de la mujer conforme a las normas masculinas, forzándolas a simular un rol 
abnegado, "espiritual" e idealizado al extremo. Una incorporeidad que suponía la renuncia a su condición humana, la absoluta deshumanización. Rescatar los deseos de las trobairitz y sus reivindicaciones supuso, por tanto, un esfuerzo doblemente extenuante: el rechazo al rol de musa y la recuperación de la voz en primera persona. No olvidemos, como señala D. Aguilera que "le lieu de la poésie est un espace d'expression pour la trobairitz en tant que femme" (2012: 179). Un espacio, este, que el Dolce Stil Novo aniquiló desde el concepto de "musa" como donna angelicata.

Los poetas estilnovistas pusieron en boca de sus amadas angelicales todo un reguero de afirmaciones y actitudes enaltecidas e, incluso, despóticas. Las convirtieron en seres inalcanzables y deshumanizados. Quizá consideraron que ello satisfacía a las mujeres de su tiempo. La belleza estética de sus poesías es innegable. Es la paradoja de la recepción de estas obras literarias ocho siglos después.

\section{REFERENCIAS BIBLIOGRÁFICAS}

Aguilera, Delphine. 2012. Femmes poètes du Moyen-Âge. Paris: L'Harmattan.

Alvar, Carlos, ed. 1984. El Dolce Stil Novo. 47 sonetos y 3 canciones (Antología). Madrid: Visor.

Arriaga Flórez, Mercedes, Daniele Cerrato y María Rosal Nadales. 2012. Poetas italianas de los siglos XIII y XIV en la Querella de las mujeres. Sevilla: Arcibel Editores.

Bertelli, Italo. 1983. La poesia di Guido Guinizzelli e la poetica del 'Dolce Stil Nuovo'. Firenze: Le Monnier.

Bogin, Meg. 1978. Les femmes troubadours. Paris: Denoë1/Gonthier.

Boyer, Agustín. 1989. Estudio descriptivo del Libro de las virtuosas e claras mugeres de don Alvaro de Luna: fuentes, género y ubicación en el debate feminista del siglo $X V$. University of California, Berkeley. Tesis Doctoral.

Contini, Gianfranco. 1960. Poeti del Duecento. Vol. 2, Tomos I-II. Milano-Napoli: Riccardo Ricciardi Editore.

Creixell Vidal-Quadras, Inés, ed. 1985. Andreas Capellanus. De Amore. Barcelona: El Festín de Esopo.

De Maio, Romeo. 1988. Mujer y Renacimiento. Madrid: Mondadori.

Dronke, Peter. 1995. La lírica en la Edad Media. Barcelona: Ariel.

Gagliardi, Antonio. 2003. Guinizzelli, Dante, Petrarca. L'inquietudine del poeta. Torino: Edizione dell'Orso.

Gil Esteve, Manuel y Rovira Soler, Matilda, eds. 1997. Dante Alighieri De Vulgari Eloquentia. Madrid: Palas Atenea.

González Ruiz, Nicolás, ed. 1980. Dante Alighieri. Obras completas. Madrid: BAC. $4^{\text {a }}$ ed.

Huizinga, Johan. 1985. El otoño de la Edad Media. Madrid: Alianza Editorial.

Le Goff, Jacques. 1990. Los intelectuales en la Edad Media. Barcelona: Gedisa. $2^{\mathrm{a}}$ ed. 
Marti, Mario. 1969. Poeti del Dolce Stil Nuovo. Firenze: Le Monnier.

Martinengo, Marirì. 1997. Las trovadoras, poetisas del amor cortés. Madrid: horas y HORAS.

Martínez de Merlo, Luis. 1988. Tres poetisas italianas del Renacimeinto. Vittoria Colonna, Gaspara Stampa, Chiara Mastraini. Madrid: Hiperión.

Niccoli, Ottavia. 1993. La mujer del Renacimiento. Madrid: Alianza Editorial.

Oroz Reta, José y Marcos Casquero, Manuel A., eds. 2004. San Isidoro de Sevilla, Etimologías. Madrid: Biblioteca de Autores Cristianos.

Pelosi, Pietro. 1998. Guido Guinizzelli. Rime. Napoli: Liguori Editore.

Pirovano, Donato y Grimaldi, Marco, eds. 2015. Nuova edizione commentata delle Opere di Dante. Vita Nuova. Rime. Vol. 1-T. I. Roma: Salerno Editrice.

Pozuelo Yvancos, José María. 1988. Teoría del lenguaje literario. Madrid: Cátedra.

Rieger, Angelica. 1991. Trobairitz. Der Beitrag der Frau in der altokzitanischen höfischen Lyrik. Edition des Gesamtkorpus. Tübingen: Max Niemeyer Verlag.

Riquer, Martín de. 1983. Los trovadores. Historia literaria y textos, 3 vols. Barcelona: Ariel.

Riquer, Isabel de. 1997. "Las trobairitz provenzales en el fin de siglo". Lectora 3: 27-37. Rossi, Luciano. 2004. Ripartiamo da Guinizzelli. En Brugnolo, Furio y Peron, Gianfelice, eds. Da Guido Guinizzelli a Dante. Nuove prospettive sulla lirica del Duecento. Padova: Il Poligrafo. 25-58.

Ruiz Doménec, José Enrique. 1990. La mujer que mira (crónicas de la cultura cortés). Barcelona: Sirmio.

Salinas, Pedro. 1981. Jorge Manrique o tradición y originalidad. Barcelona: Sex Barral. $2^{\mathrm{a}}$ ed.

Santidrián, Pedro R. y Manuela Astruga, eds. 1993. Cartas de Abelardo y Eloísa. Madrid: Alianza Editorial.

Vàrvaro, Alberto. 1983. Literatura románica de la Edad Media. Barcelona: Ariel.

Víñez Sánchez, Antonia. 2013. "La voz disidente de las trobairitz." En Navarrete Navarrete, María Teresa y Soler Gallo, Miguel, (eds.). El eterno presente de la literatura. Estudios literarios de la Edad Media al siglo XIX. Roma: Aracne Editrice. 35-63.

Víñez Sánchez, Antonia. 2016. "La Donna Stella de Guido Guinizzelli a la luz del arcano XVII 'La Estrella' del Tarot”. En Ramello, Laura; Borio, Alex e Nicola, Elisabetta, (eds.). "Par estude ou par acoustumance". Saggi offerti a Marco Piccat. Alessandria: Edizioni dell'Orso. 671-687.

Víñez Sánchez, Antonia. 2017. "Si bei`s dizon tuich que mout descove que dompna prei a cavallier de se. Cortesía y anti-cortesía en la poesía de Castelloza". En Cerrato, Daniele, (ed.). Desde los márgenes: narraciones y representaciones femeninas. Sevilla: Benilde. 271-286.

Víñez Sánchez, Antonia y Sáez Durán, Juan. 2018. "Los precedentes de la 'Querelles des Femmes' en la poesía románica medieval: las trobairitz”. En Cerrato, Daniele; Schembari, Andrea y Velázquez García, Sara, (eds.). 'Querelle des Femmes': Male and Female voices in Italy and Europe. Poland: volumina.pl. 11-26. 